\title{
HYDROGEOPHYSICAL MAPPING WITH THE TRANSIENT ELECTROMAGNETIC SOUNDING METHOD.
}

\author{
L.H. Poulsen\# and N.B. Christensen* \\ (\# Geological Survey of Denmark and Greenland, GEUS, Thoravej 8, \\ DK-2400 Copenhagen NV, Denmark, \\ * Department of Earth Sciences, Aarhus University, Finlandsgade 8, \\ DK-8200 Aarhus N, Denmark)
}

\section{INTRODUCTION}

The transient electromagnetic sounding method is an effective reconnaissance exploration tool for mapping good conductors like clay and salt water. In the Bredebro area in Southern Jutland, Denmark, the method has been used for mapping an aquifer situated in a Tertiary valley system eroded out in heavy clay. The area was previously mapped with gravity measurements and this investigation indicated the presence of a Tertiary valley system. The transient electromagnetic investigation has confined the location of the Tertiary valley found in the gravity investigation and added more information about the boundaries of the valley system.

The measuring strategy for the TEM survey was based on the earlier gravity investigation and transient sounding were made on four profiles across the positive residual Bourguer anomaly. It was decided to measure with approximately $100 \mathrm{~m}$ between every TEM sounding on the profile lines not to miss important information about the valley system.

\section{THE TEM SURVEY}

Inversion of the TEM soundings is performed with a computer based least squares iterative inversion and analysis program SELMA (Simultaneous Electromagnetic Layered Modeling and Analysis, Christensen and Auken, 1992). The basic model is a onedimensional plane parallel earth model consisting of homogeneous and transversely isotropic layers.

The standard inversion is performed with as few layers as possible, three to five, and results generally in a good fit to data. Besides finding the depth to the good conductor the inversion gives the average resistivity of basic geological formations. The results of the inversions with few-layer models are presented as a contoured map of the depth to the bottom of the valley and as concatenated 1D models on the profile lines, see figure $1 \mathrm{a}$ ). Each layer is represented as a block. The block thickness is identical to the interpreted layer thickness, and a sounding is assumed to represent the area half way to the next sounding on the profile line. On the model sections the Tertiary valley system is indicated by the presence of high resistivities above the good conductor.

To estimate the uncertainty of the determination of resistivities and depths a standard deviation model section is developed. These sections show the standard deviation of the model parameters along the profile, figure $1 \mathrm{~b}$ ). A standard deviation between 0 and 0.2 indicates well-determined parameters, a value between 0.2 and 0.5 means reasonable to poor determination, and 0.5 to 1.0 is a poor determination. The numbers at the layer boundaries are the standard deviation of the depth to the layer. 
Beside the standard inversion a quasi-continuous interpretation with multiple-layer models is performed. The idea with multiple-layer interpretations is to interpret transient electromagnetic soundings without an initial model for the iterative interpretation. The multi-layer model has fixed layer boundaries and "soft bounds" between the layer resistivities to ensure a proper smoothness constaint. The model has asymptotically seven layers per decade, which is about four layers per decade in measured time. There are between 14 and 17 layers in the model. The different numbers of layers is due to changes in the topographic level (elevation above mean sea level) from sounding to sounding. All layer boundaries beneath the level of the lowest sounding are in the same elevation. Layer boundaries in identical levels ensure that the contouring of the profiles does not depend on the location of the layer boundaries but on the distribution of the resistivity/conductivity. The thickness of the top layers in the models is between 1 and 4 meters. Figure $1 \mathrm{c}$ ) shows the contoured multiple-layer resistivity sections for profile 1 . The gray scale indicates the resistivities of the layers. The model sections shows the distribution of high and low resistivity in profiles across the tertiary valley and thereby the location of the valley system.

From looking at the resistivity of the layered structures it is possible to say something about the width of the valley. On the two resistivity model sections a geological interpretation is sketched. In the geological interpretation it is important to remember that the standard inversion with few-layer models gives average resistivities in the interpreted layers and that the multiple-layer models give a smoothed picture of the distribution of the conductivity in the earth. From the resistivity model sections and the standard deviation model sections a geological interpretation has been carried out (figure 1d). Two- and threedimensional effects (Auken, 1995) and equivalence (Fitterman, Meekes \& Ritsema, 1988), in transient electromagnetic data, have been kept in mind in the geological interpretation, where layer boundaries do not always coincide with the $1 \mathrm{D}$ interpretation.

\section{THE GEOLOGICAL INTERPRETATION}

In the Geological interpretation a boundary in the good conductor is marked. The good conductor is interpreted as tertiary mica clay and the boundary is assumed to reflect changes in the conductivity due to changes in the clay contents. This interpretation of the Tertiary clay matches a borehole description, but the boundary does not match a geological boundary. The drilling is the only deep drilling in the area.

The layer marked as "top layer" has an undefined thickness and resistivity and it can be anything from aeolic sand to top soil. Beneath the top layer there is quaternary diluvial wet or dry sand. The layer may contain moraine benches and thin clay layers. On profile 1 the filling in the-valley has a lower resistivity than the layer on top. Geologically it is interpreted as quaternary wet sand with a variable silt contents. On the eastern side of profile 1 a small depression filled with sediments which have a resistivity of $17 \mathrm{Ohmm}$ is seen. This depression is only seen on one transient electromagnetic sounding, and as such it is an unreliable feature.

\section{GRAVITY MODELING OF THE TEM RESULTS}

A renewed gravity modeling has been carried out with geometrical constraints from the TEM survey, see figure 2 . The grid size of the gravity modeling is $50 \mathrm{~m}$ horizontal times $25 \mathrm{~m}$ vertical. The model has been given an anomalous mass of $0.15 \mathrm{~g} / \mathrm{cm}^{\wedge} 3$ for the valley fillings, $0.06 \mathrm{~g} / \mathrm{cm}^{\wedge} 3$ for the top layer in the Tertiary clay, 0.06 for the small depression and $0 \mathrm{~g} / \mathrm{cm}^{\wedge} 3$ for the rest of the layers. The gravity response of the model based on the transient electromagnetic profile does not match the gravity data exactly. The difference between the gravity response and the measured gravity data must be ascribed to the simplified models. 


\section{Profile 1}

a)

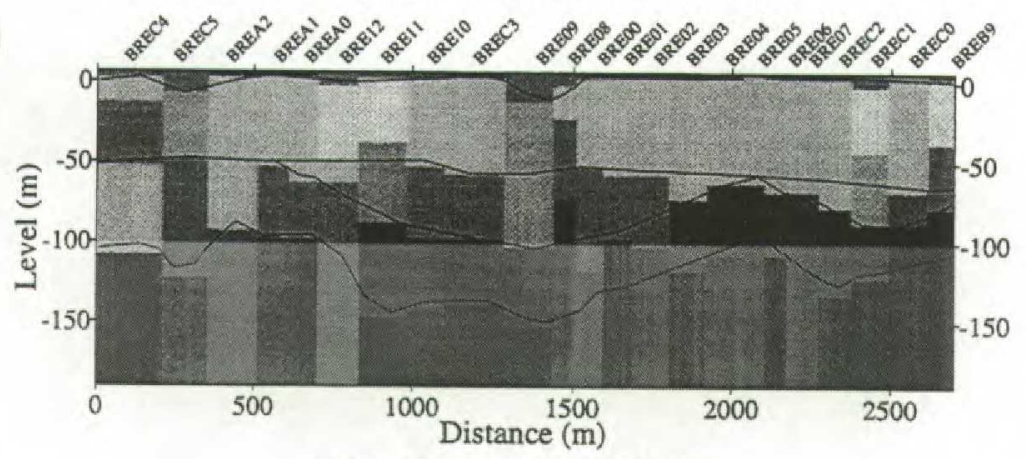

Few-layer Resistivity Model Section

b)

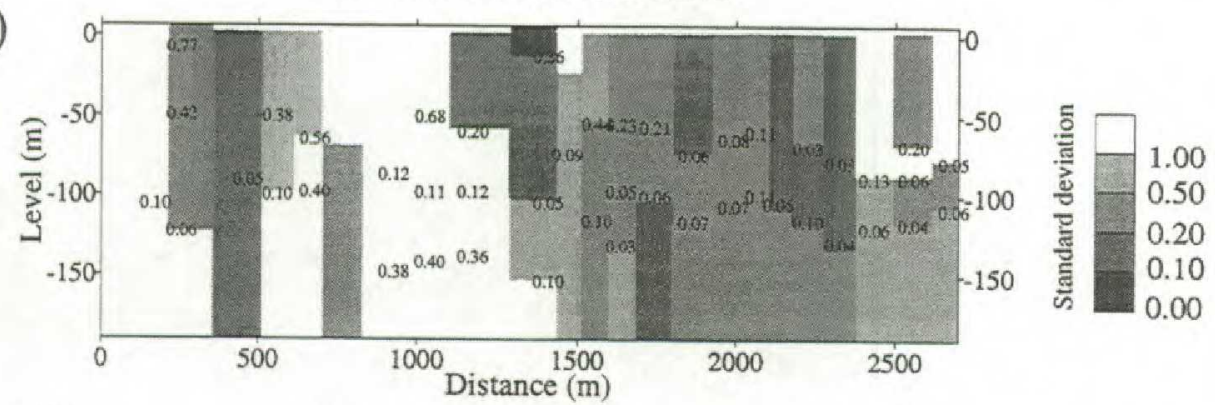

UTM $\quad$ Multiple-layer Model Section $\quad$ UTM

c)
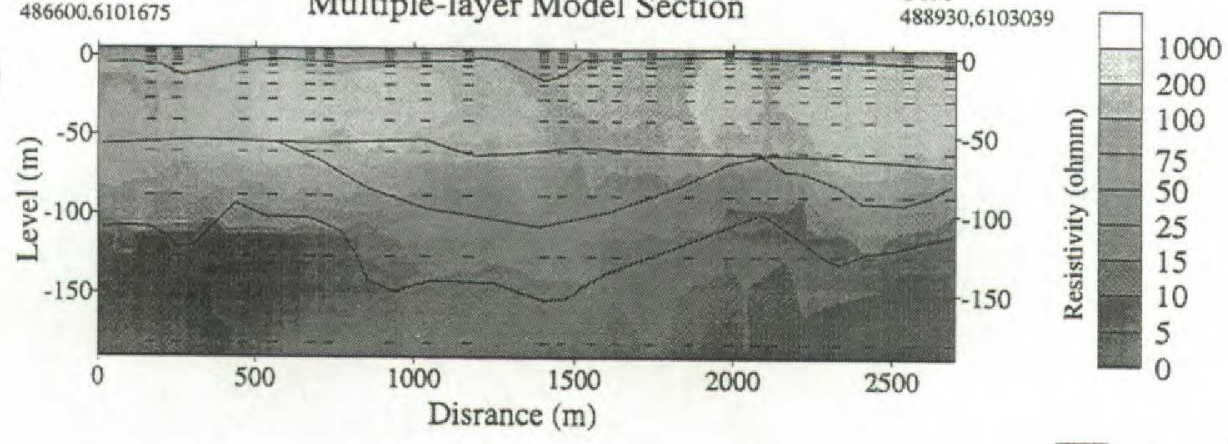

d)

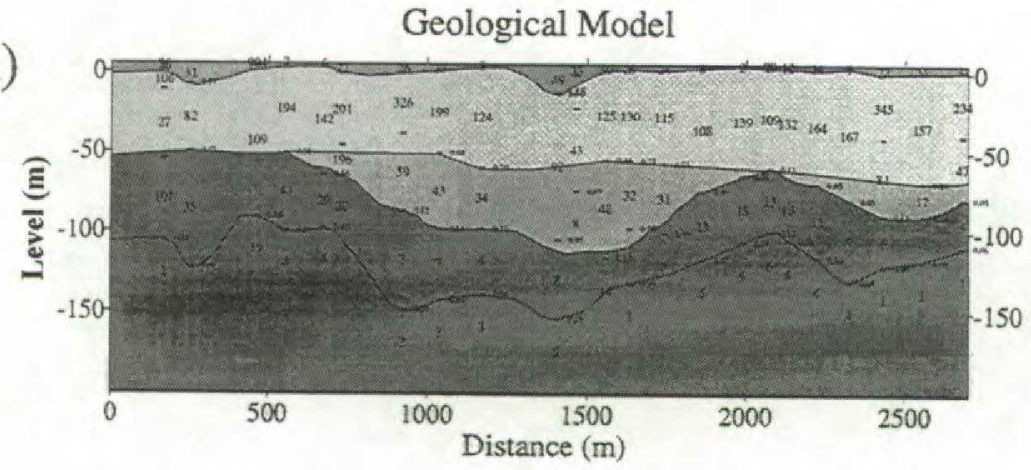

Top layer.

Quaternary diluvial sand (dry and wet), with moraine layers.

Quaternary diluvial sand (wet).

\section{Moraine deposit} Saale glaciation.

Tertiary mica clay.

Figure 1. Model sections of profile 1. a) is the resistivity model section from few-layer interpretation. The block thickness is identical to the interpreted layer thickness. The gray scale of the blocks represent the resistivity of the layer. b) shows the standard deviation of the few layer interpretations. The block gray scale represents the standard deviation of the layer resistivity and the numbers at the layer boundaries are the standard deviation of the depth to the layer. c) is the model section from multiple-layer models and shows the distribution of the conductivity in the predefined layers. High resistivity at depth shows the location of the valley system. d) is the interpreted geological model. 
The investigation resulted in a detailed picture of the geology of the target area, which can be used for hydrogeological groundwater modeling and determination of a drilling strategy for groundwater abstraction. The results demonstrate the usefulness of transient electromagnetic profile data in mapping Tertiary valley systems. In geological interpretation the model sections are very powerful tools, they show the resistivity, the depth and the position of the valley system very clearly. The inversion techniques are robust and give the standard deviation of the model parameters which is very important in the estimation of the reliability of the model and the model sections.

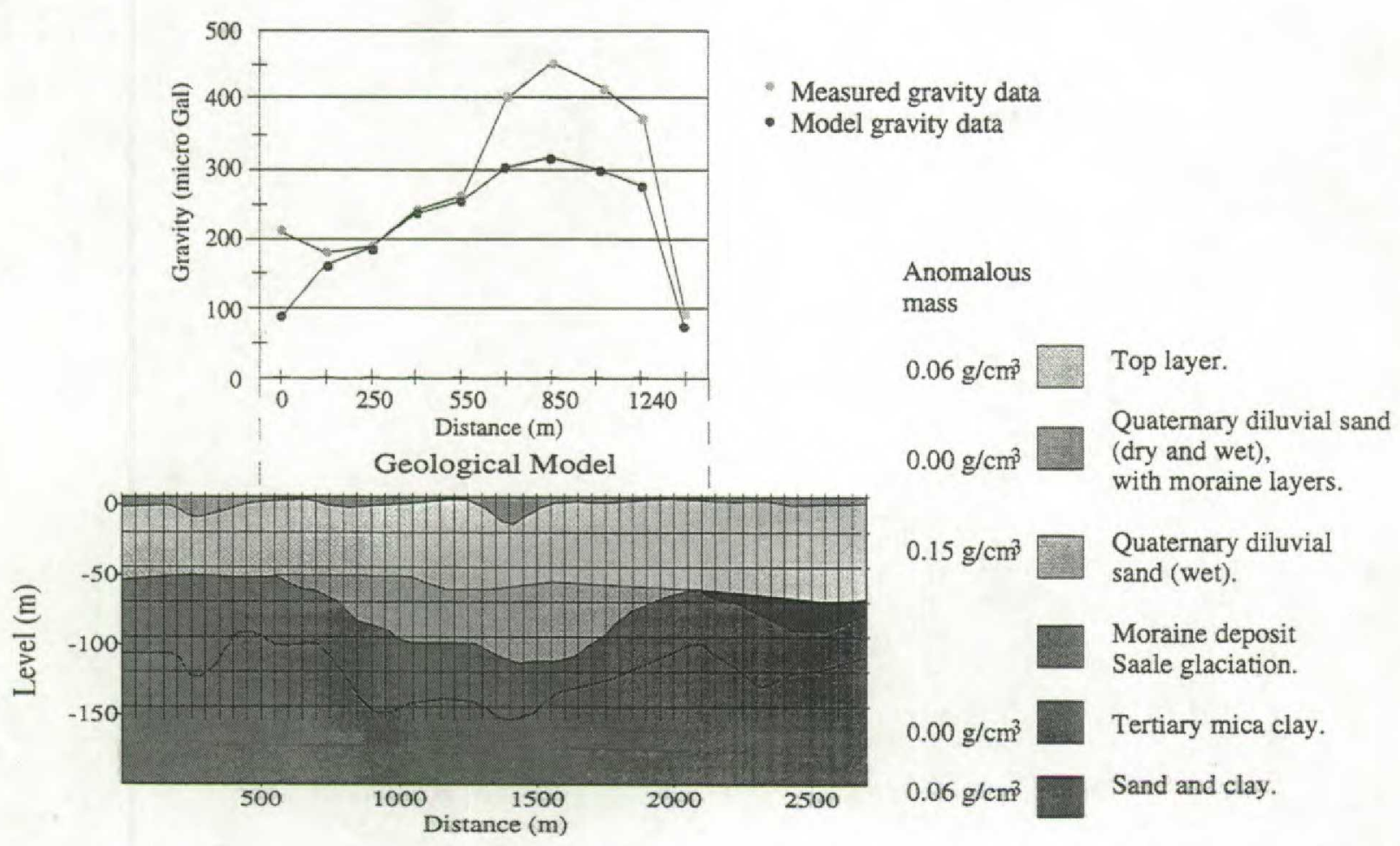

Figure 2. A very simple gravity model of profile 1 . The grid size of the gravity modeling is $50 \mathrm{~m}$ horizontal time $25 \mathrm{~m}$ vertical. The anomalous masses is shown on the figure. The gravity response of the model based on the transient electromagnetic profile does not match the gravity data exactly.

\section{REFERENCES}

Auken, E., 1995, 1D Time Domain Electromagnetic Interpretations over 2D/3D Structures. Proceeding of the Symposium on the Application of Geophysical to Engineering and Environmental Problems, April 1995, Orlando, Florida, p 329-338

Christensen,N.B. \& Auken, E., 1992, Simultaneous electromagnetic layered model analysis. In: Jacobsen, B.H. (ed), Proceedings of the Interdisciplinary Inversion Workshop 1, Geoskrifter 41, Aarhus University, P 49-56.

Fitterman, D.V., Meekes, J.A.C. \& Ritsema, I.L., 1988, Equivalence behavior of three electrical soundong methods as applied to hydrogeological problems,Presented at EAEG, the Netherlands, $37 \mathrm{p}$. 